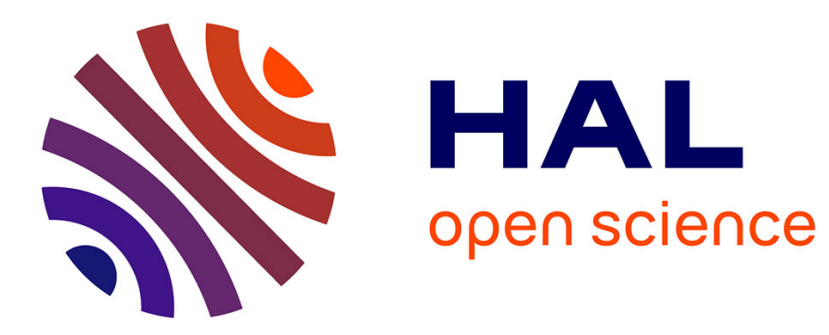

\title{
Analysis of a geometrical multiscale blood flow model based on the coupling of ODE's and hyperbolic PDE's
}

\author{
Miguel Angel Fernández, Vuk Milisic, Alfio Quarteroni
}

\section{To cite this version:}

Miguel Angel Fernández, Vuk Milisic, Alfio Quarteroni. Analysis of a geometrical multiscale blood flow model based on the coupling of ODE's and hyperbolic PDE's. [Research Report] RR-5127, INRIA. 2004. inria-00071456

\section{HAL Id: inria-00071456 \\ https://hal.inria.fr/inria-00071456}

Submitted on 23 May 2006

HAL is a multi-disciplinary open access archive for the deposit and dissemination of scientific research documents, whether they are published or not. The documents may come from teaching and research institutions in France or abroad, or from public or private research centers.
L'archive ouverte pluridisciplinaire HAL, est destinée au dépôt et à la diffusion de documents scientifiques de niveau recherche, publiés ou non, émanant des établissements d'enseignement et de recherche français ou étrangers, des laboratoires publics ou privés. 
INSTITUT NATIONAL DE RECHERCHE EN INFORMATIQUE ET EN AUTOMATIQUE

\title{
Analysis of a geometrical multiscale blood flow model based on the coupling of ODE's and hyperbolic PDE's
}

\author{
Miguel Ángel Fernández — Vuk Milišić — Alfio Quarteroni
}

\section{$\mathbf{N}^{\circ} 5127$}

Février 2004

THÈME 4 



\title{
Analysis of a geometrical multiscale blood flow model based on the coupling of ODE's and hyperbolic PDE's
}

\author{
Miguel Ángel Fernández*, Vuk Milišić ${ }^{*}$, Alfio Quarteroni ${ }^{\ddagger}$ \\ Thème 4 - Simulation et optimisation \\ de systèmes complexes \\ Projet BANG
}

Rapport de recherche $n^{\circ} 5127$ - Février $2004-29$ pages

\begin{abstract}
For the numerical simulation of the circulatory system, geometrical multiscale models based on the coupling of systems of differential equations with different spatial dimensions is becoming common practice. In this work we address the mathematical analysis of a coupled multiscale system involving a zero-dimensional model, describing the global characteristics of the circulatory system, and a one-dimensional model giving the pressure propagation along a straight vessel. We provide a local-in-time existence and uniqueness of classical solutions for this coupled problem. To this purpose we reformulate the original problem in a general abstract framework by splitting it into subproblems (the 0D system of ODE's and the 1D hyperbolic system of PDE's), then, we use fixed-point techniques. The abstract result is then applied to the original blood flow case under very realistic hypotheses on the data. This work represents the $1 \mathrm{D}-0 \mathrm{D}$ counterpart of the 3D-0D mathematical analysis reported in [SIAM J. on Multiscale Model. Simul., 1(2) (2003) 173].
\end{abstract}

Key-words: multiscale modelling, hyperbolic systems, lumped parameters models, blood flow modelling, fixed-point techniques

* INRIA-BANG / EPFL-IACS, email: miguel.fernandez@inria.fr

$\dagger$ EPFL-IACS, email: vuk.milisic@epfl.ch

$\ddagger$ EPFL-IACS, email: alfio.quarteroni@epfl.ch 


\section{Analyse d'un modèle multi-échelle pour les écoulements sanguis basé dans le couplage d'EDOs et d'EDPs hyperboliques}

Résumé : L'utilisation de modèles multi-échelle, couplant des systèmes d'équations différentielles avec différentes dimensions d'espace, est devenue une approche usuelle dans la simulation numérique du système cardiovasculaire. Dans ce travail on s'intéresse à l'analyse mathématique d'un système couplé multi-échelle constitué d'un modéle $0 \mathrm{D}$, décrivant les caractéristiques globales du système cardiovasculaire, et d'un modèle $1 \mathrm{D}$, tenant compte de la propagation des ondes de pression. Pour ce problème on fournit un résultat local (en temps) d'existence et d'unicité de solution régulière. Avec cet objectif, on reformule le problème original dans une cadre abstrait général en le décomposant dans deux subproblèmes (le système $0 \mathrm{D}$ d'équations différentielle ordianaires (ODE) et le système $1 \mathrm{D}$ d'équations aux dérivées partielles (EDP) hyperboliques), ensuite on utilise des techniques de point fixe. Le résultat abstrait obtenu est ainsi appliqué au cas des écoulements sanguins, avec des hypothèses sur les données très réalistes. En outre, ce travail représente l'homologue 1D-0D de l'analyse mathématique 3D-0D introduit dans [SIAM J. on Multiscale Model. Simul., 1(2) (2003) 173].

Mots-clés : modélisation multi-échelle, systèmes hyperboliques, modèles $0 \mathrm{D}$, écoulements sanguins, techniques de point fixe 


\section{Introduction}

Problems arising in the numerical modelling of the human cardiovascular system often require an accurate description of the flow in a specific sensible sub-region (carotid bifurcation, stented artery, etc.). The description of such local phenomena is better addressed by means of three-dimensional (3D) simulations, based on the numerical approximation of the incompressible Navier-Stokes equations, possibly accounting for compliant (moving) boundaries. However, from the computational point of view, numerical simulations of the whole circulatory system completely based on these equations are, at the moment, unaffordable.

To overcome this limitation, several (geometrical) multiscale approaches have recently been proposed in the literature, see e.g. $[22,21,6,3,29,19,11,4]$. The main principle consists in coupling different models with a decreasing level of accuracy, which is compensated by their decreasing computational complexity. Starting from the 3D incompressible Navier-Stokes equations, under certain assumptions, one can derive a one-dimensional (1D) model, stated in terms of hyperbolic equations along the axial coordinate of the vessel. With further simplifications, we can obtain a zero-dimensional (0D) model (or lumped parameters model), represented by a system of ordinary differential equations. One-dimensional models have shown to provide useful information at low computational cost [6], in particular, these models are very well adapted for the simulation of wave propagations in the arterial tree $[27,28,30,4]$. On the other hand, the global characteristics of the system, including specific districts as the heart or the venous bed, may be described by $0 \mathrm{D}$ models, which are essentially based on the analogy between the arterial tree and an electric network $[31,4]$.

Within this multiscale framework, different kind of coupled models have been proposed in the literature, in particular, the coupling between 3D and $1 \mathrm{D}$ models reported in [3] and that of $3 \mathrm{D}$ and $0 \mathrm{D}$ analysed in $[6,11]$. The mathematical theory for the coupling between 0D models and 3D models (without wall compliance) was carried out in [24]. Yet, a heterogeneous coupling involving a $1 \mathrm{D}$ description of the arterial tree and $0 \mathrm{D}$ description of the peripheral circulation and the heart has been addressed in $[7,4,29]$, in blood flow engineering.

The aim of the present paper is to provide a rigorous mathematical analysis of the heterogeneous 1D-0D coupling, reported in [4], involving non-linear systems of hyperbolic PDE's and ODE's. We provide a local-in-time existence and uniqueness result of classical solutions. To this purpose we give our original problem a general and abstract framework by splitting it into subproblems (the 0D system of ODE's and the 1D hyperbolic system of PDE's). Thus, using standard fixed-point techniques [32, 24], we are able to prove our main theorem. Then, under very realistic hypotheses on the data, we apply it to the original blood flow case. In this sense, our analysis requires a partial extension of the work in [2] to the case of non-monotone boundary conditions on the 1D model. On the other hand, this work represents the 1D-0D counterpart of the 3D-0D mathematical analysis reported in [24].

The outline of this paper is as follows. In section 2 we introduce the $1 \mathrm{D}$ model, some basic features of lumped parameter models leading to OD systems of ODE's, and then we formulate the coupled $1 \mathrm{D}-0 \mathrm{D}$ problem. In section 3 , we introduce the main result of this 
Miguel Ángel Fernández, Vuk Milišić, Alfio Quarteroni

paper, namely, the existence and uniqueness of solutions for an abstract coupled problem involving a general system of ODE's and a hyperbolic $2 \times 2$ system. As the main motivation of this work is the blood flow, this result is then rigorously applied to the specific case of the blood flow. Section 4 provides a detailed proof of our main result. In order to illustrate the coupled problem, we show a numerical evidence in section 5. Finally, in section 6 we present some conclusions and perspectives related to this work.

\section{Problem setting}

In this section we recall briefly the basic $1 \mathrm{D}$ non-linear hyperbolic model for the description of a single cylindrical straight arterial element, and the general lumped parameter description of the cardiovascular system. Then, we address the way to couple together the two systems. The mathematical analysis of the resulting coupled model is the main purpose of this work.

\subsection{The 1D-model}

One-dimensional hyperbolic models have been used by many authors to study various issues related to the vascular system, see for instance $[30,19,29,5,27,28,2]$. They are derived from the assumption that single tracts of an artery can be approximated by a straight compliant channel. Their simplicity yields a clear advantage over more complex models especially for real-time computations when quick answers are needed.

These models can be derived from the incompressible Navier-Stokes equations assuming that the flow is axi-symmetric. Indeed, supposing that the ratio between the radius and length of the vessel is small, one can average the equations over the cross-section. Then, assuming a specific velocity profile given as a function of the cross-sectional average velocity and the radius $[29,2]$, one obtains the following system of two partial differential equations

$$
\left\{\begin{array}{l}
\frac{\partial A}{\partial t}+\frac{\partial Q}{\partial x}=0, \quad x \in(0, l), \quad t>0 \\
\frac{\partial Q}{\partial t}+\frac{\partial}{\partial x}\left(\frac{\alpha Q^{2}}{A}\right)+\frac{A}{\rho} \frac{\partial p}{\partial x}=-K_{r} \frac{Q}{A}, \quad x \in(0, l), \quad t>0
\end{array}\right.
$$

The unknowns are the cross-section area $A$, the flow rate $Q$, the cross-section averaged pressure $p$. Moreover, $\alpha$ stands for the kinetic energy coefficient, $x$ denotes the axial direction, $\rho$ the blood density and $K_{r}$ is related to the velocity profile assumed in the vessel. If a Poiseuille profile is assumed (which is of course a simplification), $K_{r}=8 \pi \rho \nu$, where $\nu$ is the kinematic blood viscosity (see [8] for other possible values).

In spite of its simple form, this system is nonetheless capable of predicting very accurately the wave propagation phenomena inside the arterial system. To close system (1), we introduce the following constitutive law for the pressure:

$$
p=\frac{\beta}{A_{0}}\left(\sqrt{A}-\sqrt{A_{0}}\right), \quad \beta=\frac{\sqrt{\pi} h E}{\left(1-\sigma^{2}\right)},
$$


where $A_{0}$ is the constant cross-section at rest, $E, h$ and $\sigma$ the Young modulus, the thickness and Poisson coefficients of the vessel wall respectively. Expression (2) entails that the pressure is a linear function for the vessel radius [20,22].

At this point, we make the following assumptions: the constant $K_{r}$ is set to zero, the convective effects are dominant, and the source term is one order of magnitude lower than the inertial term see [2], for instance. Finally, the kinetic energy coefficient $\alpha$ is set equal to 1 ; this means assuming a flat profile inside the vessel, which is reasonable for straight vessels in blood flow $[2,8]$. Thus, system (1) reduces to the following quasi-linear hyperbolic system

$$
\left\{\begin{array}{l}
\frac{\partial A}{\partial t}+\frac{\partial Q}{\partial x}=0, \quad x \in(0, l), \quad t>0, \\
\frac{\partial Q}{\partial t}+\frac{\partial}{\partial x}\left(\frac{Q^{2}}{A}\right)+\frac{A}{\rho} \frac{\partial p}{\partial x}=0, \quad x \in(0, l), \quad t>0 .
\end{array}\right.
$$

In non-conservative form, system (3) reads

$$
\frac{\partial U}{\partial t}+H(U) \frac{\partial U}{\partial x}=0
$$

with the notations

$$
U=\left[\begin{array}{l}
A \\
Q
\end{array}\right], \quad H(U)=\left[\begin{array}{cc}
0 & 1 \\
\frac{\beta}{2 \rho A_{0}} \sqrt{A}-u^{2} & 2 u
\end{array}\right], \quad u=\frac{Q}{A} .
$$

Assuming that the cross-section area $A$ remains positive, the matrix $H$ can be diagonalised, namely, $H=R \Lambda L$, where

$$
\Lambda=\left[\begin{array}{ll}
\lambda & 0 \\
0 & \mu
\end{array}\right], \quad L=\left[\begin{array}{ll}
-\mu & 1 \\
-\lambda & 1
\end{array}\right], \quad R=L^{-1}
$$

The eigenvalues $\lambda$ and $\mu$ are given by [8]

$$
\lambda=u-c, \quad \mu=u+c, \quad c=\sqrt{\frac{\beta}{2 \rho A_{0}}} A^{\frac{1}{4}},
$$

where $u$ is the axial velocity and $c$ the sound speed. The system can be rewritten in diagonal form as

$$
\left\{\begin{array}{l}
\frac{\partial z}{\partial t}+\lambda(w, z) \frac{\partial z}{\partial x}=0 \\
\frac{\partial w}{\partial t}+\mu(w, z) \frac{\partial w}{\partial x}=0
\end{array}\right.
$$

where we have denoted by $z$ and $w$ the following Riemann invariants (or characteristic variables)

$$
z=4 c-u, \quad w=4 c+u
$$


Miguel Ángel Fernández, Vuk Milišić, Alfio Quarteroni

Owing to (5), we can express the eigenvalues in terms of Riemann invariants as follows

$$
\mu=\frac{5}{8} w-\frac{3}{8} z, \quad \lambda=\frac{3}{8} w-\frac{5}{8} z .
$$

The physical unknowns $p$ and $Q$ can be expressed themselves as functions of the characteristic variables:

$$
\begin{aligned}
p & =2 \rho c^{2}-\frac{\beta}{\sqrt{A_{0}}}=2 \rho\left(\frac{w+z}{8}\right)^{2}-\frac{\beta}{\sqrt{A_{0}}}, \\
Q & =\frac{2 A_{0}^{2} \rho^{2}}{\beta^{2}}(w-z)\left[\frac{w+z}{8}\right]^{4} .
\end{aligned}
$$

The diagonal formulation (6) suggests the correct boundary treatment.

If, for instance, one has at disposal the time history $q(t)$ of a physical variable $\phi=$ $\phi(A, Q)$, as for instance $A$ or $Q$ or even $Q / A$, the boundary condition

$$
\phi(A(t), Q(t))=q(t), \quad \forall t \in I, \quad \text { at } x=0,
$$

is admissible under certain restrictions [23, 26, 14], which in our case reduce to exclude the case where $\phi$ may be expressed solely as function of $z$. In particular, the imposition of either average pressure (cross-section area) or mass flux are both admissible. So imposing a given pressure $p_{\mathrm{g}}$ on the boundary, implies that the two characteristic variables are related to each other. Indeed, from (9), at $x=0$, we impose

$$
2 \rho\left(\frac{w(0, t)+z(0, t)}{8}\right)^{2}-\frac{\beta}{\sqrt{A_{0}}}=p_{\mathrm{g}} .
$$

If $A$ remains strictly positive, $w+z$ keeps a constant sign. Thus, we can explicitly obtain the incoming characteristic variable $w$ as a function of $p_{\mathrm{g}}$ and the outgoing characteristic variable $z$, i.e.,

$$
w(0, t)=8\left[\frac{1}{2 \rho}\left(p_{g}+\frac{\beta}{A_{0}}\right)\right]^{\frac{1}{2}}-z(0, t)=8 c-z(0, t) .
$$

Since $z$ is constant along the outgoing characteristic curve, $z(0, t)$ only depends on the initial condition $z_{0}$. Therefore, we only prescribe the value of $w$ on the boundary. The boundary condition at one end of the domain depends on the outgoing characteristic variable. If the domain is bounded, say $0 \leq x \leq l$, there exists a critical time, say $t_{\mathrm{c}}$, sufficiently large beyond which this variable depends on the values of the other end point as well. $t_{\mathrm{c}}$ is larger than the time at which the incoming characteristic curve at $x=0$ hits the boundary $x=l$ (see figure 1). Using a symmetric argument, we can deduce that $t_{\mathrm{c}}$ is also greater than the time at which the characteristic curve starting from $(x, t)=(l, 0)$ reaches $(x, t)=(0,0)$. We confine our analysis to a time interval $\left[0, T_{\max }\right]$ small enough so that the above mentioned characteristic curves do not cross (see figure 1 and refer to [13] for a similiar approach). In this case it is obvious that $T_{\max }<t_{\mathrm{c}}$ 

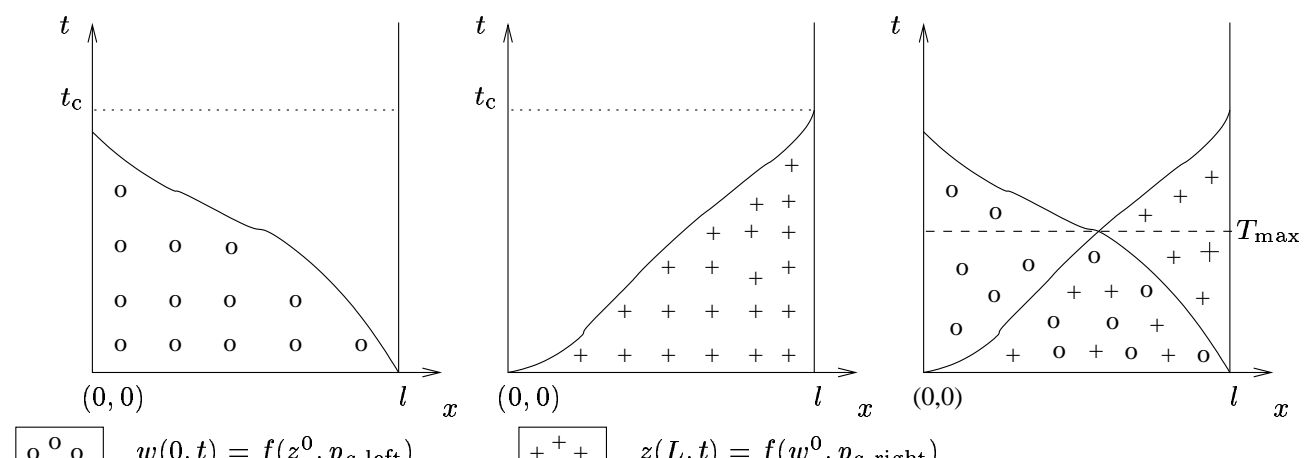

$\mathrm{o}^{\mathrm{o}} \mathrm{o} \quad w(0, t)=f\left(z^{0}, p_{g, \text { left }}\right)$

$+^{+}+\quad z(L, t)=f\left(w^{0}, p_{g, \text { right }}\right)$

Figure 1: Subdomains where the Riemann invariants depend only on the initial data and a single boundary condition

\subsection{Lumped parameters model}

The most important vessels and branches of the cardiovascular system can be described by patching together several components based on the one-dimensional model introduced in the previous paragraph, $[27,28,30,19]$. In particular, in [27] several numerical simulations of a network composed by 55 arteries are reported. This approach has, however, two main drawbacks:

1. it cannot reflect a salient feature of the cardiovascular system, i.e. its inter-dependency nature which derives from being a "closed system". Indeed, dealing with one-dimensional (or even three-dimensional) models requires the prescription of specific (and accurate) data on the artificial boundaries delimitating the computational domain [30, 27, 19].

2. a realistic description of complex terminal districts of the circulatory system (heart, capillary bed, venous circulation,... ) can not be achieved by hyperbolic one-dimensional models $[8$, Chapter 2]).

These difficulties can be overcome, through the introduction of lumped parameters models. The latter are expressed in terms of systems of ordinary differential equations, describing the averaged (in time) mass and flow rate in a specific terminal compartment of the circulatory system, see for instance $[8,31,10,17]$. Since they do not account for variations in space, they are often called zero-dimensional models. They can be derived starting from the onedimensional system (1) by integrating the equations in space. Indeed, after linearization, we obtain $[8,31])$ :

$$
\left\{\begin{array}{l}
C \frac{\mathrm{d} \hat{p}}{\mathrm{~d} t}+Q_{2}-Q_{1}=0, \quad t>0, \\
L \frac{\mathrm{d} \hat{Q}}{\mathrm{~d} t}+R \hat{Q}+P_{2}-P_{1}=0, \quad t>0 .
\end{array}\right.
$$


Miguel Ángel Fernández, Vuk Milišić, Alfio Quarteroni

Here, $\hat{p}$ and $\hat{Q}$ stand for the mean pressure and flow-rate over the whole compartment, respectively, with

$$
Q_{1}(t)=Q(0, t), \quad P_{1}(t)=P(0, t), \quad Q_{2}(t)=Q(l, t), \quad P_{2}(t)=P(l, t) .
$$

The parameters $R, L$ and $C$ denote resistance, inductance and capacitance, and they depend on the physical and geometrical properties of the pipe [8, 31]. Precisely, for a Poiseuille flow one can set

$$
R=\frac{8 \rho \nu l}{\pi r_{0}^{4}}, \quad L=\frac{\rho l}{\pi r_{0}^{2}}, \quad C=\frac{3 \pi r_{0}^{3} l}{2 E h},
$$

where $r_{0}$ stands for the vessel's radius. In order to close mathematically (11) we need some further assumptions. Let us suppose that some upstream and downstream data are available, for instance $Q_{1}$ and $P_{2}$. It is also reasonable to approximate the unknowns on the upstream and downstream sections with the state variables, i.e. $\hat{p} \approx P_{1}$ and $\hat{Q} \approx Q_{2}$. If the length of the vessel is small enough this assumption makes sense and acts like a discretization error. Moreover in blood flow the wave speed is about $\left(1\right.$ to $10 \mathrm{~m} \cdot \mathrm{s}^{-1}$ ), so that the information propagates almost instantaneously from two neighbor points in a single vessel (whose length might be of few centimeters).

Under these additional assumptions, (11) can be written as

$$
\frac{\mathrm{d} \mathbf{y}}{\mathrm{d} t}=A \mathbf{y}+\mathbf{b}, \quad t>0
$$

where

$$
\mathbf{y}=\left[\begin{array}{c}
P_{1} \\
Q_{2}
\end{array}\right], \quad A=\left[\begin{array}{cc}
0 & -\frac{1}{C} \\
\frac{1}{L} & -\frac{R}{L}
\end{array}\right], \quad \mathbf{b}=\left[\begin{array}{c}
\frac{Q_{1}}{C} \\
\frac{P_{2}}{L}
\end{array}\right] .
$$

System (12) can be equivalently regarded as the mathematical description of an electric circuit which is known as $\mathcal{L}$-network. In fact, in this hydraulic/electric analogy, pressure and flow rate correspond to the electric voltage and current, the resistance $R$ is related to the blood viscosity, the inductance $L$ to the blood inertia and the capacitance $C$ to the wall compliance. More details can be found in [8].

Equation (12) represents, in abstract form, a lumped parameters description of the blood flow in a compliant cylindrical vessel, involving the mean values of the flow rate and the pressure in this domain, as well as the upstream and downstream flow rate and pressure. However, as mentioned above, a specific description is required for more complex systems such as the heart, which provides the energy necessary to blood circulation in the whole system. Heart can be considered as a couple of pumps. In particular, in [8], each ventricle is represented as a compliant vessel whose compliance $C(t)$ changes in time. On the other hand, in order to describe the heart functionality, in the electric/hydraulic analogy every valve is modelled by a diode for the current according to the value of the applied voltage drop $[8,4]$. 
From these considerations, the whole circulation can be therefore described by means of a system of differential equations whose abstract form reads

$$
\frac{\mathrm{d} \mathbf{y}}{\mathrm{d} t}=A \mathbf{y}+\mathbf{r}_{\mathrm{H}}(\mathbf{y}, t)+\mathbf{b}(\mathbf{y}, t), \quad t>0 .
$$

Here, $\mathbf{y} \in \mathbb{R}^{m}$ stands for the vector of state variables, $A \in \mathbb{R}^{m \times m}$ is a matrix, $\mathbf{r}_{\mathrm{H}}(\mathbf{y}, t) \in \mathbb{R}^{m}$ is a vector whose dependence on $\mathbf{y}$ and $t$ should be ascribed to the presence of valves and heart's ventricles compliances $[25,8,21]$. The vector $\mathbf{b}(\mathbf{y}, t) \in \mathbb{R}^{m}$ represents a generalized source term which provides external data to the system. Specific instances of (13) can be found, for example, in $[24,6,4]$.

\subsection{Coupling 1D and lumped parameters model}

The simulation of blood flow in the circulatory system can be made by resorting to multiscale models which couple three-dimensional, one-dimensional and zero-dimensional systems. These three paradigms feature a (decreasing) level of accuracy, which is however compensated by a decreasing level of computational complexity. In previous papers different kind of coupled models have been considered. We recall the coupling between 3D and 1D models in [6], that of $1 \mathrm{D}$ and $0 \mathrm{D}$ models in $[7,4]$. The mathematical theory for the coupling between $0 \mathrm{D}$ models and 3D models (without wall compliance) is carried out in [24]. In this paper we investigate existence and uniqueness of the solution of the problem that arises from coupling of $1 \mathrm{D}$ and $0 \mathrm{D}$ models.

Indeed, we couple a system of ordinary differential equations presented in detail in the previous section with a $2 \times 2$ system of conservation laws describing section-averaged flow rate and pressure presented in section 2.1 .

As already mentioned in section 2.1 we look for the solution of the $1 \mathrm{D}$ model for a time small enough so that the characteristic curves coming from both boundaries do not intersect. This means that the closed loop coupling can be functionally replaced by the connection of two infinite 1D domains interacting through a $0 \mathrm{D}$ model.From the mathematical point of view, the analysis of this $1 \mathrm{D}-0 \mathrm{D}-1 \mathrm{D}$ coupling can be reduced to a simple 0D-1D coupling.More precisely, once provided the time $\hat{T}$ of existence of the solution of the $0 \mathrm{D}-1 \mathrm{D}$ coupling, the time of existence for the corresponding $1 \mathrm{D}-0 \mathrm{D}-1 \mathrm{D}$ coupling is given by $\min \left(T_{\max }, \hat{T}\right)$.

The coupling between the $0 \mathrm{D}$ and $1 \mathrm{D}$ models is achieved by imposing the continuity of pressure and flow-rate at the interface(refer to $[6,4]$, for instance). More precisely, the given pressure $p_{\mathrm{g}}$ in paragraph 2.1 for the $1 \mathrm{D}$ model is provided by the $0 \mathrm{D}$ part, i.e., $p_{\mathrm{g}}$ represents here a specific entry of the state vector $\mathbf{y}$ in (13). Consequently, using (10), we impose

$$
w(0, t)=8\left[\frac{1}{2 \rho}\left(p_{\mathrm{g}}(t)+\frac{\beta}{A_{0}}\right)\right]^{\frac{1}{2}}-z(0, t)=g(\mathbf{y}(t), z(0, t)) .
$$


On the other hand, the 1D model supplies the flow-rate to the 0D model. Using (14) in (9), the flow-rate on the boundary can also be expressed, as a function of $\mathbf{y}(t)$ and $z(0, t)$., i.e.,

$$
Q(\mathbf{y}(t), z(0, t))=\frac{A_{0}^{2}}{\beta^{2}}\left(4\left[\frac{1}{2 \rho}\left(p_{\mathrm{g}}(t)+\frac{\beta}{A_{0}}\right)\right]^{\frac{1}{2}}-z(0, t)\right)\left[p_{\mathrm{g}}(t)+\frac{\beta}{A_{0}}\right]^{2} .
$$

We point out that this value appears in (13) as part of the source term $\mathbf{b}$.

REMARK 2.1. The choice of imposing the pressure as a boundary condition for the $1 D$ model and the flow-rate as a source term for the 0D part is an arbitrary choice. Indeed, changing the structure of the $0 D$ network connected to the $1 D$ vessel, one could obtain the symmetric exchange of data.

In summary, the coupled 1D-0D problem can be set in the following abstract framework: find $\mathbf{y}:[0, T] \rightarrow \mathbb{R}^{m}$ and $z, w: \mathbb{R}^{+} \times[0, T] \rightarrow \mathbb{R}$ such that

$$
\left\{\begin{array}{l}
\frac{\partial w}{\partial t}+\mu(w, z) \frac{\partial w}{\partial x}=0, \quad \text { in } \quad \mathbb{R}^{+} \times[0, T] \\
\frac{\partial z}{\partial t}+\lambda(w, z) \frac{\partial z}{\partial x}=0, \quad \text { in } \quad \mathbb{R}^{+} \times[0, T] \\
z(x, 0)=z_{0}(x), \quad x \in \mathbb{R}^{+} \\
w(x, 0)=w_{0}(x), \quad x \in \mathbb{R}^{+}, \\
w(0, t)=g(\mathbf{y}(t), z(0, t)), \quad t \in \mathbb{R}^{+} \\
\frac{\mathrm{d} \mathbf{y}}{\mathrm{d} t}=\mathcal{G}(\mathbf{y}, z(0, t), t), \quad \text { in } \quad \mathbb{R}^{+} \\
\mathbf{y}(0)=\mathbf{y}_{0}
\end{array}\right.
$$

Here, $g$ is given by (14) and $\mathcal{G}$ by

$$
\mathcal{G}(\mathbf{y}, z, t)=A \mathbf{y}+\mathbf{r}_{\mathrm{H}}(\mathbf{y}, t)+\mathbf{b}(Q(\mathbf{y}(t), z))
$$

with $Q$ defined by (15).

In the following sections we provide a rigorous mathematical analysis for problem (16).

\section{An abstract existence and uniqueness result}

In this section we state our main result that is an existence and uniqueness theorem for the coupled problem (16). To analyse the strong solutions of this system, we introduce the following hypotheses on the data:

(H1) (i) There exists an open subset $\mathcal{E} \subset \mathbb{R}^{2}$ such that $\lambda$, $\mu$ and their derivatives are Lipschitz continuous functions in $\mathcal{E}$ and

$$
\lambda(w, z)<\mu(w, z), \quad \forall(w, z) \in \mathcal{E} .
$$

i.e., the system is strictly hyperbolic.

INRIA 
(ii) The eigenvalues $\lambda$ and $\mu$ satisfy

$$
\frac{\partial \lambda}{\partial z}<0, \quad \frac{\partial \mu}{\partial w}>0, \quad \text { in } \mathcal{E}
$$

(H2) The initial data $z_{0}$ and $w_{0}$ belongs to $C^{1}\left(\mathbb{R}^{+}\right) \cap L^{\infty}\left(\mathbb{R}^{+}\right)$and $\left(w_{0}(x), z_{0}(x)\right) \in \mathcal{E}$ for all $x \in \mathbb{R}^{+}$, moreover $z_{0}^{\prime} \leq 0$ and $w_{0}^{\prime} \geq 0$ in $\mathbb{R}^{+}$.

(H3) There exists $\gamma>0$ such that $g \in C^{1}\left(B_{\mathbb{R}^{m}}(0, \gamma) \times \mathbb{R}^{+}\right), B_{\mathbb{R}^{m}}(0, \gamma)$ being a ball of radius $\gamma$ in $\mathbb{R}^{m}$

(H4) The boundary is non-characteristic; this is guaranteed, for instance, if the following sufficient condition is fulfilled

$$
\begin{aligned}
& \sup _{(x, t) \in \mathbb{R}^{+} \times[0, T]}\left\{\lambda\left(g\left(\mathbf{y}(t), z_{0}(x)\right), z_{0}(x)\right)\right\}<0, \\
& \inf _{(x, t) \in \mathbb{R}^{+} \times[0, T]}\left\{\mu\left(g\left(\mathbf{y}(t), z_{0}(x)\right), z_{0}(x)\right)\right\}>0 .
\end{aligned}
$$

(H5) The following compatibility conditions between initial and boundary data at time $t=0$ hold:

$$
\begin{aligned}
w_{0}(0) & =g\left(\mathbf{y}_{0}, z_{0}(0)\right) \\
-\mu\left(w_{0}(0), z_{0}(0)\right) w_{0}^{\prime}(0) & =\frac{\partial g}{\partial \mathbf{y}}\left(\mathbf{y}_{0}, z_{0}(0)\right) \cdot \mathbf{y}^{\prime}(0) \\
& +\frac{\partial g}{\partial z}\left(\mathbf{y}_{0}, z_{0}(0)\right) \lambda\left(w_{0}(0), z_{0}(0)\right) z_{0}^{\prime}(0) .
\end{aligned}
$$

These identities ensure the continuity of $w(x, t)$ and $\frac{\partial w}{\partial x}$ at $(x, t)=(0,0)$.

(H6) $\mathcal{G} \in \mathbf{C}^{0}\left(\mathbb{R}^{m} \times \mathbb{R} \times \mathbb{R}\right)$ is continuous and locally Lipschitz continuous with respect to its two first arguments. In addition, we assume that $\left|\mathbf{y}_{0}\right|<\gamma$.

The following theorem represents the main result of this paper; its proof is given in section 4.

THEOREM 3.1. Suppose that hypotheses $(\mathrm{H} 1)-(\mathrm{H} 6)$ hold. Then there exists a positive time $0<\hat{T} \leq T$ such that the coupled problem (16) admits a strong solution $(\mathbf{y}, w, z) \in$ $\mathbf{C}^{1}[0, \hat{T}] \times C^{1}\left(\mathbb{R}^{+} \times[0, \hat{T}]\right)^{2}$.

\subsection{Analysis of the coupled 1D-0D problem (16)}

In this paragraph we aim at applying the main theorem 3.1 to the specific case of the coupling described in section 2.3 and summarized by the system (16). For that we need to verify the hypotheses $(\mathrm{H} 1)-(\mathrm{H} 6)$. 
Miguel Ángel Fernández, Vuk Milišić, Alfio Quarteroni

The local Lipschitz property of the right hand side of (13) is satisfied because the elements used to construct the electric network (as explained in section 2.3) provide locally Lipschitz terms with respect to the unknowns. The interface condition, being essentially a polynomial relating the pressure $p$ to the outgoing characteristic $z(0, t)$ (see formula (15)) is also locally Lipschitz.

Proposition 3.2. Suppose that $z_{0}$ is a bounded function of $x$ such that

$$
z_{0, \min } \leq z_{0}(x) \leq z_{0, \max }, \quad \forall x \in \mathbb{R}^{+},
$$

Moreover, we make the following assumptions on the boundary data $p(t)$,

$$
\begin{aligned}
& 2 \rho\left[\frac{z_{0, \max }}{5}\right]^{2}-\frac{\beta}{\sqrt{A_{0}}}<p(t)<2 \rho\left[\frac{z_{0, \min }}{3}\right]^{2}-\frac{\beta}{\sqrt{A_{0}}}, \quad \forall t \in[0, T] \\
& p(t)>-\frac{\beta}{\sqrt{A_{0}}},
\end{aligned}
$$

then $\lambda(w(0, t), z(0, t))<0<\mu((0, t), z(0, t))$ and $A(0, t)>0$ and assumption (H4) holds.

Proof. To show that (17) implies $\lambda<0<\mu$ we use the expressions (7) and (8) to set:

$$
\lambda=\frac{3}{8} w-\frac{5}{8} z=3 c-\frac{3}{8} z-\frac{5}{8} z .
$$

Here we use the boundary condition (10), since $p<2 \rho\left[\frac{z_{0, \max }}{3}\right]^{2}-\frac{\beta_{0}}{\sqrt{A_{0}}}$ we obtain $\lambda<0$. Similarly,

$$
\mu=\frac{5}{8} w-\frac{3}{8} z=5 c-\frac{3}{8} z-\frac{5}{8} z,
$$

which is positive due to the first inequality of (17).

To see that the second expression of $(17)$ implies $A(0, t)>0$ it suffices to write

$$
\frac{\beta}{A_{0}} \sqrt{A}=p+\frac{\beta}{\sqrt{A_{0}}}>0,
$$

that directly gives the condition that $p$ has to satisfy.

In order to satisfy $(\mathrm{H} 1)_{(\mathrm{i})}$ we apply the following result already established in [2],

THEOREM 3.3. Suppose that the left boundary $x=0$ is non-characteristic (i.e. $\lambda(0, t)<$ $0<\mu(0, t))$. If $A(x, 0)>0$ for all $x$, and if $A(0, t)>0$ on the left boundary, then $A(x, t)>0, \forall(x, t) \in \mathbb{R}^{+} \times[0, T]$, and so the system is strictly hyperbolic in $\mathbb{R}^{+} \times[0, T]$.

This theorem proves the strict hyperbolicity of the system under our hypotheses on the boundary data and initial data (hypothesis $(\mathrm{H} 2)$ and (H3)) . Indeed, in hypothesis $(\mathrm{H} 1)_{(\mathrm{i})}$, we can take

$$
\mathcal{E}=\left\{(w, z) \in \mathbb{R}^{2} / w+z>0\right\} .
$$

This provides bounds on imposed data $p_{\mathrm{g}}$ (a single entry of the state vector $\mathbf{y}$ in (16)) with respect to the initial condition $z_{0}$. 
To fulfill the hypothesis $(\mathrm{H} 2)$ on the initial conditions $\left(z_{0}, w_{0}\right)$ we require that

$$
z_{0}^{\prime}(x) \leq 0, \quad w_{0}^{\prime}(x) \geq 0, \quad z_{0}(x) \geq z_{0}, \quad w_{0}(x) \leq w_{0, \max }, \quad w_{0}(x)+z_{0}(x)>0,
$$

for all $x \in \mathbb{R}^{+}$. The first two conditions provide global existence of solutions $(z, w)$ in regions that are not influenced by the boundary condition. The remaining three inequalities guarantee the boundedness of the data, so that (H2) is verified.

Thanks to the bounds established on the boundary data $p_{\mathrm{g}}$ we can easily verify that the derivatives of $g$ are bounded ( $p_{\mathrm{g}}$ is far enough from the point $\left.-\frac{\beta}{\sqrt{A_{0}}}\right)$ so that (H3) holds.

REMARK 3.1. In terms of the conserved quantities $A$ and $Q$, assuming, for simplicity, constant initial data, $A(0, x)=A_{0}$ and $Q(0, x)=0$, where $A_{0}$ is the cross-sectional area of the unstressed artery, condition (17) requires that the pressure prescribed on the left boundary is such that

$$
-\frac{9}{25} \frac{\beta}{\sqrt{A_{0}}}<p_{\mathrm{g}}(t)<\frac{7}{9} \frac{\beta}{\sqrt{A_{0}}}
$$

Considering that the expected variation of pressure around a reference state $p_{r}=0$ is an order of magnitude smaller than $\frac{\beta}{\sqrt{A_{0}}}$, this looks quite reasonable.

\section{Proof of Theorem 3.1}

In this section we reformulate (16) as a fixed point problem (as done in [24], for the coupling of 3D and 0D models for blood flow circulation). We show that the corresponding iteration operator $\mathcal{T}$ has a unique fixed point (see Theorem 4.7 below) using the classical Banach fixed point theorem [32, Page 17], that we quote here for reader's convenience:

THEOREM 4.1. Suppose that

(i) A given operator satisfies $\mathcal{T}: M \subseteq X \longrightarrow M$, i.e., $M$ is mapped into itself by $\mathcal{T}$;

(ii) $M$ is a closed nonempty set in complete metric space $(X, d)$ (d being the distance function);

(iii) $\mathcal{T}$ is contractive, i.e.,

$$
d(T x, T y) \leq k d(x, y),
$$

for all $x, y \in M$ and for a fixed $k$ such that $0 \leq k<1$. Then $\mathcal{T}$ has a unique fixed point in $M$, that is a point $x \in M$ such that $\mathcal{T}(x)=x$.

$\mathrm{RR} \mathrm{n}^{\circ} 5127$ 


\subsection{The iteration operator $\mathcal{T}$}

For $\sigma>\left\|z_{0}\right\|_{C^{0}\left(\mathbb{R}^{+}\right)}, \gamma>0$ (given by hypothesis (H3)) and $\xi>0$ (to be fixed later, see Lemma 4.2) we introduce the following subsets

$$
\begin{aligned}
& B_{C^{0}[0, T]}(\sigma)=\left\{u \in C^{0}[0, T] /\|u\|_{C^{0}[0, T]} \leq \sigma\right\}, \\
& B_{\mathbf{C}^{0}[0, T]}(\gamma)=\left\{\mathbf{y} \in \mathbf{C}^{0}[0, T] /\|\mathbf{y}\|_{\mathbf{C}^{0}[0, T]} \leq \gamma\right\}, \\
& B_{\mathbf{C}^{1}[0, T]}(\xi)=\left\{\mathbf{y} \in \mathbf{C}^{1}[0, T] / \quad\|\mathbf{y}\|_{\mathbf{C}^{1}[0, T]} \leq \xi\right\} .
\end{aligned}
$$

On the one hand, we introduce the operator

$$
\begin{aligned}
\mathcal{L}_{0 \mathrm{D}}: B_{C^{0}[0, T]}(\sigma) & \longrightarrow \mathbf{C}^{1}[0, T] \\
u & \longmapsto \mathbf{y}=\mathcal{L}_{0 \mathrm{D}}(u),
\end{aligned}
$$

where $\mathbf{y}$ is the solution of the $0 \mathrm{D}$ subproblem

$$
\left\{\begin{array}{l}
\frac{\mathrm{d} \mathbf{y}}{\mathrm{d} t}=\mathcal{G}(\mathbf{y}, u(t), t), \quad \text { in } \quad[0, T] \\
\mathbf{y}(0)=\mathbf{y}_{0} .
\end{array}\right.
$$

On the other hand, we introduce the operator

$$
\begin{aligned}
\mathcal{L}_{1 \mathrm{D}}: B_{\mathbf{C}^{0}[0, T]}(\gamma) \cap B_{\mathbf{C}^{1}[0, T]}(\xi) \subset \mathbf{C}^{1}[0, T] & \longrightarrow C^{0}[0, T] \\
\mathbf{y} & \longmapsto v=\mathcal{L}_{1 \mathrm{D}}(\mathbf{y}),
\end{aligned}
$$

where $v(t)=z(0, t)$, and $(w, z)$ is the solution of the following 1D subproblem (extracted from (16)):

$$
\left\{\begin{array}{l}
\frac{\partial w}{\partial t}+\mu(w, z) \frac{\partial w}{\partial x}=0, \quad \text { in } \quad \mathbb{R}^{+} \times[0, T] \\
\frac{\partial z}{\partial t}+\lambda(w, z) \frac{\partial z}{\partial x}=0, \quad \text { in } \quad \mathbb{R}^{+} \times[0, T] \\
z(x, 0)=z_{0}(x), \quad x \in \mathbb{R}^{+} \\
w(x, 0)=w_{0}(x), \quad x \in \mathbb{R}^{+}, \\
w(0, t)=g(\mathbf{y}(t), z(0, t)), \quad t \in[0, T] .
\end{array}\right.
$$

In other words, $v$ is the restriction at the interface $\{0\} \times[0, T]$ of the characteristic variable $z$ in (19).

At this stage, and assuming that

$$
\mathcal{L}_{0 \mathrm{D}}\left(B_{C^{0}[0, T]}(\sigma)\right) \subset B_{\mathbf{C}^{0}[0, T]}(\gamma) \cap B_{\mathbf{C}^{1}[0, T]}(\xi),
$$

we can introduce the composite operator $\mathcal{T}=\mathcal{L}_{1 \mathrm{D}} \circ \mathcal{L}_{0 \mathrm{D}}$, i.e.,

$$
\begin{aligned}
\mathcal{T}: B_{C^{0}[0, T]}(\sigma) \subset C^{0}[0, T] & \longrightarrow C^{0}[0, T] \\
u & \longmapsto v=\mathcal{T}(u)=\mathcal{L}_{1 \mathrm{D}}\left(\mathcal{L}_{0 \mathrm{D}}(u)\right)
\end{aligned}
$$


Each fixed point of $\mathcal{T}$ defines a solution of the coupled problem (16). Indeed, if $\mathcal{T}(u)=u$, then $\mathbf{y}=\mathcal{L}_{0 \mathrm{D}}(u)$ satisfies subproblem (18). Moreover, since $u=\mathcal{L}_{1 \mathrm{D}}(\mathbf{y})$, it follows that $u(t)=z(0, t)$ with $(w, z)$ satisfying (19). Hence, $\mathbf{y}$ solves

$$
\left\{\begin{array}{l}
\frac{\mathrm{d} \mathbf{y}}{\mathrm{d} t}=\mathcal{G}(\mathbf{y}, z(0, t), t), \quad \text { in }[0, T], \\
\mathbf{y}(0)=\mathbf{y}_{0},
\end{array}\right.
$$

so that $(\mathbf{y}, w, z)$ is a solution of (16). In order to apply Theorem 4.1 to the operator $\mathcal{T}$, we need some preliminary abstract results.

\subsection{Preliminary abstract results}

In this part we analyse the operators $\mathcal{L}_{1 \mathrm{D}}$ and $\mathcal{L}_{0 \mathrm{D}}$, formally defined in the previous paragraphs.

\subsubsection{The 0D operator: $\mathcal{L}_{\mathrm{OD}}$}

The following classical result guarantees that the $0 \mathrm{D}$ operator $\mathcal{L}_{0 \mathrm{D}}$ is well defined.

LEMma 4.2. Suppose that hypothesis (H6) holds and that we are given

$$
u \in B_{C^{0}[0, T]}(\sigma) .
$$

Then there exists a positive time $T_{0 \mathrm{D}}=T_{0 \mathrm{D}}(\sigma, \gamma) \leq T$ such that problem (18) admits a unique solution $\mathbf{y} \in \mathbf{C}^{1}\left[0, T_{0 \mathrm{D}}\right]$ and

$$
\begin{aligned}
\|\mathbf{y}\|_{\mathbf{C}\left[0, T_{0 D}\right]} & \leq \gamma, \\
\|\mathbf{y}\|_{\mathbf{C}^{1}\left[0, T_{0 D}\right]} & \leq \xi,
\end{aligned}
$$

where $\xi$ is a positive constant independent of $u$.

Proof. Since $\mathcal{G}$ is Lipschitz continuous, there exists a positive time $0<T_{0}(\sigma) \leq T$ such that problem (18) admits a unique solution $\mathbf{y} \in \mathbf{C}^{1}\left[0, T_{0}\right]$ (see, for instance, [1]. Integrating (18) over $[0, t]$ with $t \leq T_{0}$ and denoting by $L$ the Lipschitz constant of $\mathcal{G}$, we get

$$
\begin{aligned}
|\mathbf{y}(t)| & \leq\left|\mathbf{y}_{0}\right|+\int_{0}^{t}|\mathcal{G}(\mathbf{y}(s), u(s), s)| \\
& \leq\left|\mathbf{y}_{0}\right|+\int_{0}^{t}|\mathcal{G}(\mathbf{0}, u(s), s)|+L \int_{0}^{t}|\mathbf{y}(s)|,
\end{aligned}
$$

Thus, applying Gronwall's Lemma, we obtain

$$
|\mathbf{y}(t)| \leq\left(\left|\mathbf{y}_{0}\right|+\int_{0}^{t}|\mathcal{G}(\mathbf{0}, u(s), s)|\right) e^{L t}
$$


Now, since $\left|\mathbf{y}_{0}\right|<\gamma$ (due to hypothesis (H6)), it is possible to verify that there exists a positive time $T_{0 \mathrm{D}}=T_{0 \mathrm{D}}(\sigma, \gamma) \leq T_{0}$ such that the following inequality,

$$
\left(\left|\mathbf{y}_{0}\right|+\int_{0}^{t}|\mathcal{G}(\mathbf{0}, u(s), s)|\right) \leq \gamma e^{-L t}
$$

holds for any $0 \leq t \leq T_{0 \mathrm{D}}$. Thus, using (21), we get

$$
|\mathbf{y}(t)| \leq \gamma, \quad \forall t \in\left[0, T_{0 \mathrm{D}}\right]
$$

which gives $(20)_{1}$. On the other hand, $(20)_{2}$, can be easily derived from $(18)_{1},(20)_{1}$ and the continuity of $\mathcal{G}$.

The following comparison result ensures the continuity of the operator $\mathcal{L}_{0 \mathrm{D}}$.

LEMma 4.3. Assume that hypothesis (H6) holds, and let $\mathbf{y}_{1}$ and $\mathbf{y}_{2}$ be the solutions of (18) with respective data $u_{1}$ and $u_{2}$ in $B_{C^{0}\left[0, T_{0 \mathrm{D}}\right]}(\sigma)$, respectively, keeping the same initial condition $\mathbf{y}_{0}$. Then the following estimate holds

$$
\left\|\mathbf{y}_{1}-\mathbf{y}_{2}\right\|_{\mathbf{C}^{1}\left[0, T_{0 \mathrm{D}}\right]} \leq L T_{0 \mathrm{D}}\left\|u_{1}-u_{2}\right\|_{C^{0}\left[0, T_{0 \mathrm{D}}\right]},
$$

with $L$ the Lipschitz constant of $\mathcal{G}$.

Proof. For any $t \in\left[0, T_{0 \mathrm{D}}\right]$ we set

$$
\begin{aligned}
& \mathbf{Y}(t)=\mathbf{y}_{1}(t)-\mathbf{y}_{2}(t), \\
& U(t)=u_{1}(t)-u_{2}(t) .
\end{aligned}
$$

Then, from $(18)_{1}$ we obtain

$$
\frac{\mathrm{d} \mathbf{Y}}{\mathrm{d} t}=\mathcal{G}\left(\mathbf{y}_{1}, u_{1}(t), t\right)-\mathcal{G}\left(\mathbf{y}_{2}, u_{2}(t), t\right), \quad \forall t \in\left[0, T_{0 \mathrm{D}}\right] .
$$

Integrating this expression over $(0, t)$, using the Lipschitz property of $\mathcal{G}$, and the fact that $\mathbf{Y}(0)=0$, we get

$$
|\mathbf{Y}(t)| \leq L \int_{0}^{t}|U(s)|+L \int_{0}^{t}|\mathbf{Y}(s)|,
$$

where $L>0$ stands for the Lipschitz constant of $\mathcal{G}$. Thus, applying Gronwall's Lemma, for $t \in\left[0, T_{0 \mathrm{D}}\right]$ we obtain

$$
|\mathbf{Y}(t)| \leq\left(L \int_{0}^{t}|U(s)|\right) e^{L T} \leq L T_{0 \mathrm{D}}\|U\|_{C^{0}\left[0, T_{0 \mathrm{D}}\right]} .
$$

Moreover, from (23) using again the Lipschitz property of $\mathcal{G}$, it follows that

$$
\left|\frac{\mathrm{d} \mathbf{Y}(t)}{\mathrm{d} t}\right| \leq L(|\mathbf{Y}(t)|+|U(t)|) \mid \leq L\left(1+L T_{0 \mathrm{D}}\right)\|U\|_{C^{0}\left[0, T_{0 \mathrm{D}}\right]}, \quad \forall t \in\left[0, T_{0 \mathrm{D}}\right] .
$$

This inequality combined with (24) gives (22), which completes the proof. 


\subsubsection{The 1D operator: $\mathcal{L}_{1 \mathrm{D}}$}

In the sequel we will use the following classical result whose proof can be found in [13].

Lemma 4.4. Let $T>0$ and $f$ be a positive continuous function in $[0, T]$. Let $y_{0} \in \mathbb{R}$ and $M>0$ be such that $\left|y_{0}\right| \leq M$. Then the Cauchy problem

$$
\left\{\begin{aligned}
\frac{\mathrm{d} y}{\mathrm{~d} t}(t) & =-f(t) y^{2}(t), \quad t \in[0, T] \\
y(0) & =y_{0}
\end{aligned}\right.
$$

has a bounded solution at at least for $t \leq \hat{T}=\frac{1}{2 M\|f\|_{C^{0}[0, T]}}$.

The following result ensures that operator $\mathcal{L}_{1 \mathrm{D}}$ is well defined.

LEMma 4.5. Suppose that hypotheses $(\mathrm{H} 1)-(\mathrm{H} 5)$ hold and that we are given a vector function $\mathbf{y} \in B_{\mathbf{C}^{0}[0, T]}(\gamma) \cap B_{\mathbf{C}^{1}[0, T]}(\xi)$. Then there exists a positive time $T_{1 \mathrm{D}}(\gamma, \xi)$ such that problem (19) admits a unique strong solution

$$
(w, z) \in C^{1}\left(\Omega_{T_{1 \mathrm{D}}}\right)^{2},
$$

with $\Omega_{T_{1 \mathrm{D}}}=\mathbb{R}^{+} \times\left[0, T_{1 \mathrm{D}}\right]$. Moreover, we have the following estimates

$$
\begin{aligned}
\|z\|_{C^{0}\left(\Omega_{T_{1 D}}\right)} & \leq\left\|z_{0}\right\|_{C^{0}\left(\mathbb{R}^{+}\right)}, \\
\|z\|_{C^{1}\left(\Omega_{T_{1 \mathrm{D}}}\right)} & \leq C_{1},
\end{aligned}
$$

where $C_{1}$ is a positive constant independent of $\mathbf{y} \in B_{\mathbf{C}^{0}\left[0, T_{1 \mathrm{D}}\right]}(\gamma) \cap B_{\mathbf{C}^{1}\left[0, T_{1 \mathrm{D}}\right]}(\xi)$.

Proof. Thanks to the assumptions (H1) and (H2) we can invoke the theorem on global existence and uniqueness of strong solution, (see, e.g., [13, Page 35, Chapter 2]), to infer the existence of $C^{1}$ solution in the region

$$
D=\left\{(x, t) \in \mathbb{R}^{+} \times \mathbb{R}^{+} / \quad x \geq x_{2}(t), \quad t \geq 0\right\} .
$$

where $x_{2}$ indicates the forward characteristic issuing from the origin, see figure 2. Furthermore, according to the theorem on local existence and uniqueness of strong solution, ( [14, Page 94, Chapter 3]), by (H3), (H5) and the inequalities

$$
\lambda\left(w_{0}(0), z_{0}(0)\right)<0<\mu\left(w_{0}(0), z_{0}(0)\right),
$$

(which directly follow from (H4), by setting $t=0$ and $x=0$ ), there exists a positive time $0<T_{0} \leq T$ such that problem (19) has a unique solution $(w, z) \in C^{1}\left(D_{T_{0}}\right)^{2}$, where $D_{T_{0}}=\left\{(x, t) \in \mathbb{R}^{+} \times\left[0, T_{0}\right] / \quad 0 \leq x \leq x_{2}(t)\right\}$. Finally, using the hypotheses (H1) and (H2) we are able to explicitly provide a time $T_{1 \mathrm{D}}(\gamma, \xi) \geq T_{0}$ for the local existence that depends only on the bounds on the data of problem (19). Indeed, to this purpose, we just have to prove that, assuming that there exists a $C^{1}$ solution on the angular domain $D_{T}$ (see figure $2)$, then its $C^{1}$ norm has an upper bound.

$\mathrm{RR} \mathrm{n}^{\circ} 5127$ 


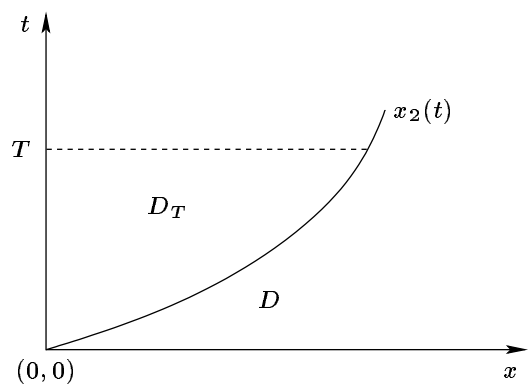

Figure 2: The domains $D$ and $D_{T}$

Using standard techniques $[13,2]$, we can derive the following estimates

$$
\begin{aligned}
\|z\|_{C^{0}\left(D_{T}\right)} & \leq\left\|z_{0}\right\|_{C^{0}\left(\mathbb{R}^{+}\right)}, \\
\|w\|_{C^{0}\left(D_{T}\right)} & \leq c_{1}\left(\gamma,\left\|z_{0}\right\|_{C^{0}\left(\mathbb{R}^{+}\right)},\left\|w_{0}\right\|_{C^{0}\left(\mathbb{R}^{+}\right)}\right) .
\end{aligned}
$$

Now we focus on the behavior of the derivatives of $(w, z)$. Using, again, standard techniques $[13,2]$ involving the Riccati's equation on the backward characteristic, we can prove the following estimate

$$
\left\|\frac{\partial z}{\partial x}\right\|_{C^{0}\left(D_{T}\right)} \leq c_{2}\left(T,\left\|z_{0}^{\prime}\right\|_{C^{0}\left(\mathrm{R}^{+}\right)}\right) .
$$

Now we derive an estimate for $\frac{\partial w}{\partial x}$. For any $(x, t) \in D_{T}$, we can track the history of $\frac{\partial w}{\partial x}$ along the forward characteristic passing through this point. Indeed, due to the hypotheses (H1) and (H4) any forward characteristic $x_{\mu}$ passing through $(x, t)$ must intersect the boundary $(x=0)$ at a unique point $(0, \alpha(x, t))$, see figure 3 .

On the other hand, on this boundary we have

$$
\begin{aligned}
\frac{\partial w}{\partial x} & =-\frac{1}{\mu(w, z)} \frac{\partial w}{\partial t} \\
& =-\frac{1}{g(\mathbf{y}(t), z), z)} \frac{\mathrm{d} g}{\mathrm{~d} t}(\mathbf{y}(t), z) \\
& =-\frac{1}{\mu}\left(\frac{\partial g}{\partial \mathbf{y}}(\mathbf{y}(t), z) \mathbf{y}^{\prime}(t)+\frac{\partial g}{\partial z}(\mathbf{y}(t), z) \frac{\partial z}{\partial t}\right) \\
& =-\frac{1}{\mu}\left(\frac{\partial g}{\partial \mathbf{y}}(\mathbf{y}(t), z) \mathbf{y}^{\prime}(t)-\lambda \frac{\partial g}{\partial z}(\mathbf{y}(t), z) \frac{\partial z}{\partial x}\right) .
\end{aligned}
$$

The dependence of $w, z$ and their derivatives on $[0, t]$ is understood. Thanks to the regularity of $g$, the bound (27) and hypotheses $(\mathrm{H} 1)_{(\mathrm{i})}$ and (H4), we obtain

$$
\left|\frac{\partial w}{\partial x}(0, t)\right| \leq c_{3}, \quad \forall t \in[0, T]
$$




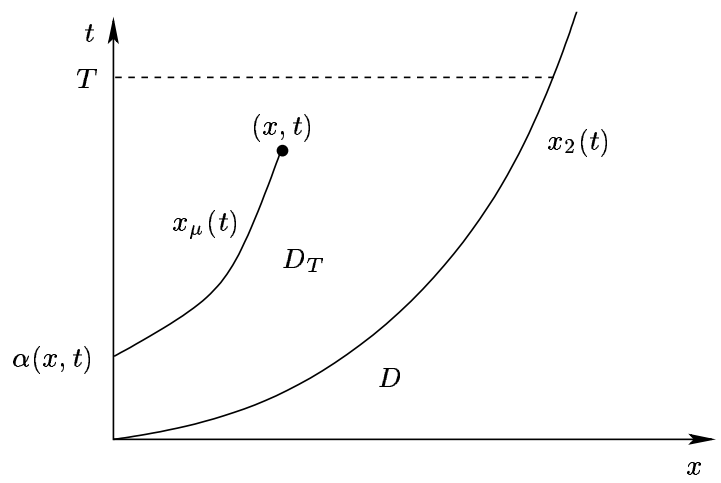

Figure 3: Tracking back the forward characteristic

where $c_{3}>0$ is positive constant depending on the boundary and initial data. We define

$$
v=e^{h(z, w)} \frac{\partial w}{\partial x}, \quad \text { in } \quad D_{T}
$$

with $h$ satisfying

$$
\frac{\partial h}{\partial z}=\frac{1}{\lambda-\mu} \frac{\partial \mu}{\partial z}, \quad \frac{\partial h}{\partial w}=0 .
$$

Along the forward characteristic $x_{\mu}, v$ satisfies the following Cauchy problem for $t \in[0, T]$

$$
\left\{\begin{array}{l}
\frac{\mathrm{d}}{\mathrm{d} s} v\left(x_{\mu}(s), s\right)=-\left(e^{-h(z, w)} \frac{\partial \mu}{\partial w} v^{2}\right)\left(x_{\mu}(s), s\right), \quad s \in[\alpha(x, t), t], \\
v(0, \alpha(x, t))=\left(e^{h(z, w)} \frac{\partial w}{\partial x}\right)(0, \alpha(x, t)) .
\end{array}\right.
$$

By setting,

$$
f(s)=\left(e^{-h(z, w)} \frac{\partial \mu}{\partial w}\right)\left(x_{\mu}(s), s\right), \quad s \in(\alpha(x, t), t),
$$

problem (30) can be written as a Riccati problem,

$$
\left\{\begin{array}{l}
\frac{\mathrm{d}}{\mathrm{d} s} v\left(x_{\mu}(s), s\right)=-f(s) v^{2}\left(x_{\mu}(s), s\right), \quad s \in[\alpha(x, t), t] \\
v(0, \alpha(x, t))=\left(e^{h(z, w)} \frac{\partial w}{\partial x}\right)(0, \alpha(x, t))
\end{array}\right.
$$

From hypothesis $(\mathrm{H} 1)_{(\mathrm{i})}$,

$$
|\lambda-\mu| \geq c_{4}>0 \quad \forall(z, w) \in \mathcal{E}
$$


so that $h$ is well defined and Lipschitz continuous. Moreover, since $\mu$ is Lipschitz continuous, $\frac{\partial \mu}{\partial z}$ is bounded. From (28), the initial condition is also bounded,

$$
|v(0, \alpha(x, t))| \leq c_{5} .
$$

Thus, it follows that $f$ is uniformly bounded in time,

$$
f(s) \leq c_{6}\left(\left\|z^{0}\right\|_{C^{0}\left(\mathbb{R}^{+}\right)},\|\mathbf{y}\|_{\mathbf{C}^{0}[0, T]}\right), \quad \forall s \in[\alpha(x, t), t] .
$$

Then, from Lemma 4.4, there exists a time

$$
T_{1 \mathrm{D}}>\frac{1}{2 c_{5} c_{6}},
$$

such that $v$ remains bounded in $D_{T_{1 \mathrm{D}}}$. Consequently, $\frac{\partial w}{\partial x}$ is also bounded, so that $(26)_{2}$ holds. Estimate (26) follows directly from $(27)_{1}$.

Lemma 4.6. Assume that hypotheses $(\mathrm{H} 1)-(\mathrm{H} 5)$ hold, and let $\left(z_{1}, w_{1}\right)$ and $\left(z_{2}, w_{2}\right)$ be the solutions of (19) corresponding, respectively, to the boundary data $\mathbf{y}_{1}$ and $\mathbf{y}_{2}$ in $B_{\mathbf{C}^{1}\left[0, T_{1 \mathrm{D}}\right]}(\gamma)$, while keeping the same initial conditions. Then

$$
w_{1}=w_{2}, \quad z_{1}=z_{2}, \quad \text { in } \quad D,
$$

and

$$
\left\|z_{1}-z_{2}\right\|_{C^{0}\left(\Omega_{T_{1 \mathrm{D}}}\right)}+\left\|w_{1}-w_{2}\right\|_{C^{0}\left(\Omega_{T_{1 \mathrm{D}}}\right)} \leq C_{2}\left\|\mathbf{y}_{1}-\mathbf{y}_{2}\right\|_{\mathbf{C}^{0}\left[0, T_{1 \mathrm{D}}\right]},
$$

where $C_{2}$ is a positive constant independent of $\mathbf{y}_{1}$ and $\mathbf{y}_{2}$.

Proof. The uniqueness of solution for the Cauchy problem [13, Remark 2.4, Chapter 2] guarantees that there exists a unique forward characteristic issuing from $x=0, t=0$ and, in particular, that (31) holds.

We set $\mathbf{Y}=\mathbf{y}_{1}-\mathbf{y}_{2}$ and

$$
\begin{aligned}
W & =w_{1}-w_{2}, \\
Z & =z_{1}-z_{2},
\end{aligned}
$$

in $D_{T_{1 \mathrm{D}}}$. Since $\left(w_{1}, z_{1}\right)$ and $\left(w_{2}, z_{2}\right)$ satisfy $(19)_{1,2}$, it follows that

$$
\begin{gathered}
\frac{\partial W}{\partial t}+\mu_{1} \frac{\partial W}{\partial x}=\left(\mu_{2}-\mu_{1}\right) \frac{\partial w_{2}}{\partial x}, \quad \text { in } \quad D_{T} \\
\frac{\partial Z}{\partial t}+\lambda_{1} \frac{\partial Z}{\partial x}=\left(\lambda_{2}-\lambda_{1}\right) \frac{\partial z_{2}}{\partial x}, \quad \text { in } \quad D_{T}
\end{gathered}
$$

where

$$
\lambda_{1}=\lambda\left(z_{1}, w_{1}\right), \quad \lambda_{2}=\lambda\left(z_{2}, w_{2}\right), \quad \mu_{1}=\mu\left(z_{1}, w_{1}\right), \quad \mu_{2}=\mu\left(z_{2}, w_{2}\right) .
$$


Multiplying (33) $)_{1}$ by $W$ and $(33)_{2}$ by $Z$ and using the Lipschitz property of the eigenvalues $\lambda$ and $\mu$ (hypothesis $(\mathrm{H} 1)_{(\mathrm{i})}$ ), one obtains

$$
\begin{gathered}
\frac{\partial W^{2}}{\partial t}+\mu_{1} \frac{\partial W^{2}}{\partial x} \leq c_{1}\left(Z^{2}+W^{2}\right), \quad \text { in } \quad D_{T} \\
\frac{\partial Z^{2}}{\partial t}+\lambda_{1} \frac{\partial Z^{2}}{\partial x} \leq c_{2}\left(Z^{2}+W^{2}\right), \quad \text { in } \quad D_{T} .
\end{gathered}
$$

Let $(x, t) \in D_{T}$. By hypothesis (H1) and (H4) the forward characteristic $x_{\mu_{1}}$ (with slope $\left.\mu_{1}\right)$ passing through $(x, t)$ must intersect the boundary $x=0$ at a unique point $(0, \alpha(x, t))$. Similarly, the backward characteristic $x_{\lambda_{1}}$ (with slope $\lambda_{1}$ ) passing through $(x, t)$ must intersect the characteristic curve $x_{2}$ at only and only on point $\left(x_{2}(\beta(x, t)), \beta(x, t)\right)$. See figure 4. Evaluating (34) 1 along $x_{\mu_{1}}$ and (34) 2 along $x_{\lambda_{1}}$ we get that for $0<t<T$,

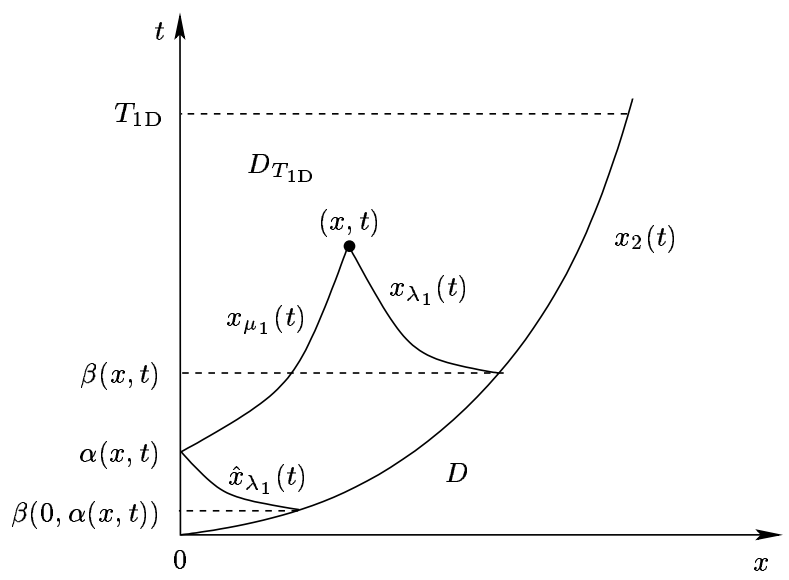

Figure 4: Tracking back the forward and backward characteristics

$$
\begin{array}{cc}
\frac{\mathrm{d}}{\mathrm{d} s} W^{2}\left(x_{\mu_{1}}(s), s\right) \leq c_{1}\left(Z^{2}+W^{2}\right)\left(x_{\mu_{1}}(s), s\right), & s \in(\alpha(x, t), t), \\
\frac{\mathrm{d}}{\mathrm{d} s} Z^{2}\left(x_{\lambda_{1}}(s), s\right) \leq c_{2}\left(Z^{2}+W^{2}\right)\left(x_{\lambda_{1}}(s), s\right), & s \in(\beta(x, t), t) .
\end{array}
$$


Integrating in time the first inequality of $(35)$ over $[\alpha(x, t), t]$,

$$
\begin{aligned}
W^{2}(x, t) \leq & W^{2}(0, \alpha(x, t))+c_{1} \int_{\alpha(x, t)}^{t}\left(Z^{2}\left(x_{\mu_{1}}(s), s\right)+W^{2}\left(x_{\mu_{1}}(s), s\right)\right) \\
= & \left|g\left(\mathbf{y}_{1}(\alpha(x, t)), z_{1}(0, \alpha(x, t))\right)-g\left(\mathbf{y}_{2}(\alpha(x, t)), z_{2}(0, \alpha(x, t))\right)\right|^{2} \\
& +c_{1} \int_{\alpha(x, t)}^{t}\left(Z^{2}\left(x_{\mu_{1}}(s), s\right)+W^{2}\left(x_{\mu_{1}}(s), s\right)\right) \\
\leq & c_{3}\left(|\mathbf{Y}(\alpha(x, t))|^{2}+Z^{2}(0, \alpha(x, t))\right) \\
& +c_{1} \int_{\alpha(x, t)}^{t}\left(Z^{2}\left(x_{\mu_{1}}(s), s\right)+W^{2}\left(x_{\mu_{1}}(s), s\right)\right),
\end{aligned}
$$

and $(35)_{2}$ over $[\beta(x, t), t]$, we get

$$
Z^{2}(x, t) \leq c_{2} \int_{\beta(x, t)}^{t}\left(Z^{2}\left(x_{\lambda_{1}}(s), s\right)+W^{2}\left(x_{\lambda_{1}}(s), s\right)\right) .
$$

Using this last inequality we obtain

$$
Z^{2}(0, \alpha(x, t)) \leq c_{1} \int_{\beta(0, \alpha(x, t))}^{\alpha(x, t)}\left(Z^{2}\left(\hat{x}_{\lambda_{1}}(s), s\right)+W^{2}\left(\hat{x}_{\lambda_{1}}(s), s\right)\right) d s,
$$

where $\hat{x}_{\lambda_{1}}$ stands for the backward characteristic (with slope $\lambda_{1}$ ) reaching the curve $x_{2}(t)$ at $\left(x_{2}(\beta(0, \alpha)), \alpha\right)$.

Here, using (37) and (38) to estimate terms depending on $Z$ in (36), we get

$$
\begin{aligned}
W^{2}(x, t)+Z^{2}(x, t) \leq & c_{3}|\mathbf{Y}(\alpha(x, t))|^{2} \\
& +c_{4} \int_{\beta(0, \alpha(x, t))}^{\alpha(x, t)}\left(Z^{2}\left(\hat{x}_{\lambda_{1}}(s), s\right)+W^{2}\left(\hat{x}_{\lambda_{1}}(s), s\right)\right) \\
& +c_{1} \int_{\alpha(x, t)}^{t}\left(Z^{2}\left(x_{\mu_{1}}(s), s\right)+W^{2}\left(x_{\mu_{1}}(s), s\right)\right) \\
& +c_{2} \int_{\beta(x, t)}^{t}\left(Z^{2}\left(x_{\lambda_{1}}(s), s\right)+W^{2}\left(x_{\lambda_{1}}(s), s\right)\right) .
\end{aligned}
$$

At this stage we define

$$
q(s)=\max _{x \in\left[0, x_{2}(s)\right]}\left\{Z^{2}(x, s)+W^{2}(x, s)\right\} .
$$


Thus, from (39) we obtain that

$$
\begin{aligned}
W^{2}(x, t)+Z^{2}(x, t) \leq & c_{3}|\mathbf{Y}(\alpha(x, t))|^{2}+ \\
& +c_{4} \int_{\beta(0, \alpha(x, t))}^{\alpha(x, t)} q(s)+c_{1} \int_{\alpha(x, t)}^{t} q(s) \\
& +c_{2} \int_{b(x, t)}^{t} q(s) \\
\leq & c_{3}\|\mathbf{Y}\|_{\mathbf{C}^{0}[0, t]}^{2}+c_{5} \int_{0}^{t} q(s) .
\end{aligned}
$$

Since this estimate holds for every $x \in\left[0, x_{2}(t)\right]$ we get

$$
q(t) \leq c_{3}\|\mathbf{Y}\|_{\mathbf{C}^{0}[0, t]}^{2}+c_{5} \int_{0}^{t} q(s) .
$$

Hence, by using Gronwall's Lemma we have

$$
q(t) \leq c_{3}\|\mathbf{Y}\|_{\mathbf{C}^{0}[0, t]}^{2} e^{c_{5} t}
$$

Consequently,

$$
\left\|z_{1}-z_{2}\right\|_{C^{0}\left(D_{T_{1 \mathrm{D}}}\right)}+\left\|w_{1}-w_{2}\right\|_{C^{0}\left(D_{T_{1 \mathrm{D}}}\right)} \leq c_{6}\left\|\mathbf{y}_{1}-\mathbf{y}_{2}\right\|_{\mathbf{C}^{0}\left[0, T_{1 \mathrm{D}}\right]} e^{c_{7} T_{1 \mathrm{D}}}
$$

which with (31) provides (32) and completes the proof. $\square$

\subsection{Properties of the operator $\mathcal{T}$}

We are therefore left to prove that the operator $\mathcal{T}$ has an unique fixed point. Indeed, the following result guarantees that the operator $\mathcal{T}$ satisfies the hypotheses of Theorem 4.1.

THEOREM 4.7. Assume that hypothesis $(\mathrm{H} 1)-(\mathrm{H} 6)$ hold. Then there exists a positive time $0<\hat{T} \leq T$ such that the operator $\mathcal{T}$ is contractive and maps $B_{C^{0}[0, \hat{T}]}(\sigma)$ into itself.

Proof. By setting $\tilde{T}=\min \left\{T_{0 \mathrm{D}}(\sigma, \gamma), T_{1 \mathrm{D}}(\gamma, \xi)\right\}$, Lemmas 4.2 and 4.5 guarantee that operator $\mathcal{T}$ is well defined. On the other hand, by combining estimate (32) of lemma 4.6 with inequality (24) from lemma 4.3, we get

$$
\begin{aligned}
\left\|\mathcal{T}\left(u_{1}\right)-\mathcal{T}\left(u_{2}\right)\right\|_{C^{0}[0, \tilde{T}]} & =\left\|\mathcal{L}_{1 D}\left(\mathcal{L}_{0 D}\left(u_{1}\right)\right)-\mathcal{L}_{1 D}\left(\mathcal{L}_{0 D}\left(u_{2}\right)\right)\right\|_{C^{0}[0, \tilde{T}]} \\
& \leq C_{2}\left\|\mathcal{L}_{0 D}\left(u_{1}\right)-\mathcal{L}_{0 D}\left(u_{2}\right)\right\|_{\mathbf{C}^{0}[0, \tilde{T}]} \\
& \leq C_{2} L \tilde{T}\left\|u_{1}-u_{2}\right\|_{C^{0}[0, \tilde{T}]}
\end{aligned}
$$

Thus, for a sufficiently small time $0<\hat{T} \leq \tilde{T}$ such that $C_{2} L \hat{T}<1$, the operator $\mathcal{T}$ is contractive. Finally, from $(26)_{1}$ and since $\sigma>\left\|z_{0}\right\|_{C^{0}\left(\mathbb{R}^{+}\right)}$we obtain that $\mathcal{T}$ maps $B_{C^{0}[0, \hat{T}]}(\sigma)$ into itself, which completes the proof. 
Setting $X=C^{0}[0, \hat{T}], M=B_{C^{0}[0, \hat{T}]}(\sigma)$ and applying Theorem 4.1 we obtain that $\mathcal{T}$ admits a unique fixed point in $B_{C^{0}[0, \hat{T}]}(\sigma)$. This claim completes the proof of Theorem 3.1.

REMARK 4.1. Although the $1 D$ operator $\mathcal{L}_{1 D}$ by itself is not contractive, (see lemma 4.6), thanks to the contractive property of the $O D$ operator $\mathcal{L}_{0 D}$, the operator $\mathcal{T}$ is contractive. $A$ similar situation occurs for the case of the 3D-0D coupling analysed in [24].

\section{A numerical test}

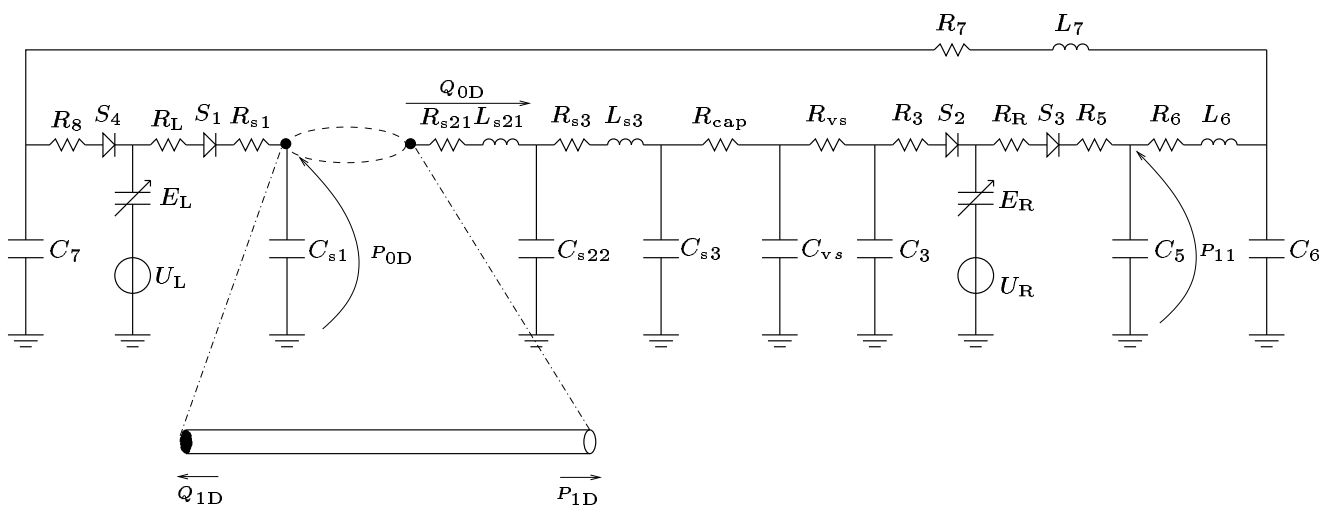

Figure 5: An example of 0D-1D coupling

Here we present the numerical solution of a 0D-1D coupling to which our existence and uniqueness results applies. We consider a lumped network describing the whole cardiovascular system including the two ventricles, the systemic tree and the lungs $[15,7]$. The complete network is represented (in compartemental form) in figure 5 . In table 1 we present the list of the different $R, L$ and $C$ coefficients used to set up the 0D model. That parameters are obtained in [16] from experiments using a mock circulatory system. In order to account for wave propagation, we have split the descending aortic compartment in two parts and we have considered the first one (defined by $R_{\mathrm{s} 21}, L_{\mathrm{s} 21}$ and $C_{\mathrm{s} 21}$ ) as a tube modelled by the $1 \mathrm{D}$ equations (1) (setting $\beta=13.3 \cdot 10^{5}$ dynes $\cdot \mathrm{cm}^{-1}$ and $A_{0}=4.91 \mathrm{~cm}^{2}$ in (2)).

By construction, the 0D model provides the pressure $P_{0 \mathrm{D}}(t)$ at the inlet and the flux $Q_{0 \mathrm{D}}$ at the outlet of the $1 \mathrm{D}$ vessel. In order to define completely our coupling, we recover as source term for the $0 \mathrm{D}$ model the flow-rate $Q_{1 \mathrm{D}}$ at the left side of the vessel and the pressure $P_{1 \mathrm{D}}$ at the right one. In figure 6 , we show the numerical results (obtained using a standard finite element Lax-Wendroff scheme, see [7, 9]), for the area and flow-rate along the $1 \mathrm{D}$ tube at different instants of a heart beat.

Figure 7 illustrates the wave forms of the left inlet pressure $Q_{0 \mathrm{D}}$, the right inlet flux $Q_{0 \mathrm{D}}$, and the pulmonary pressure $P_{11}$. The continuous line represents the coupled system while 

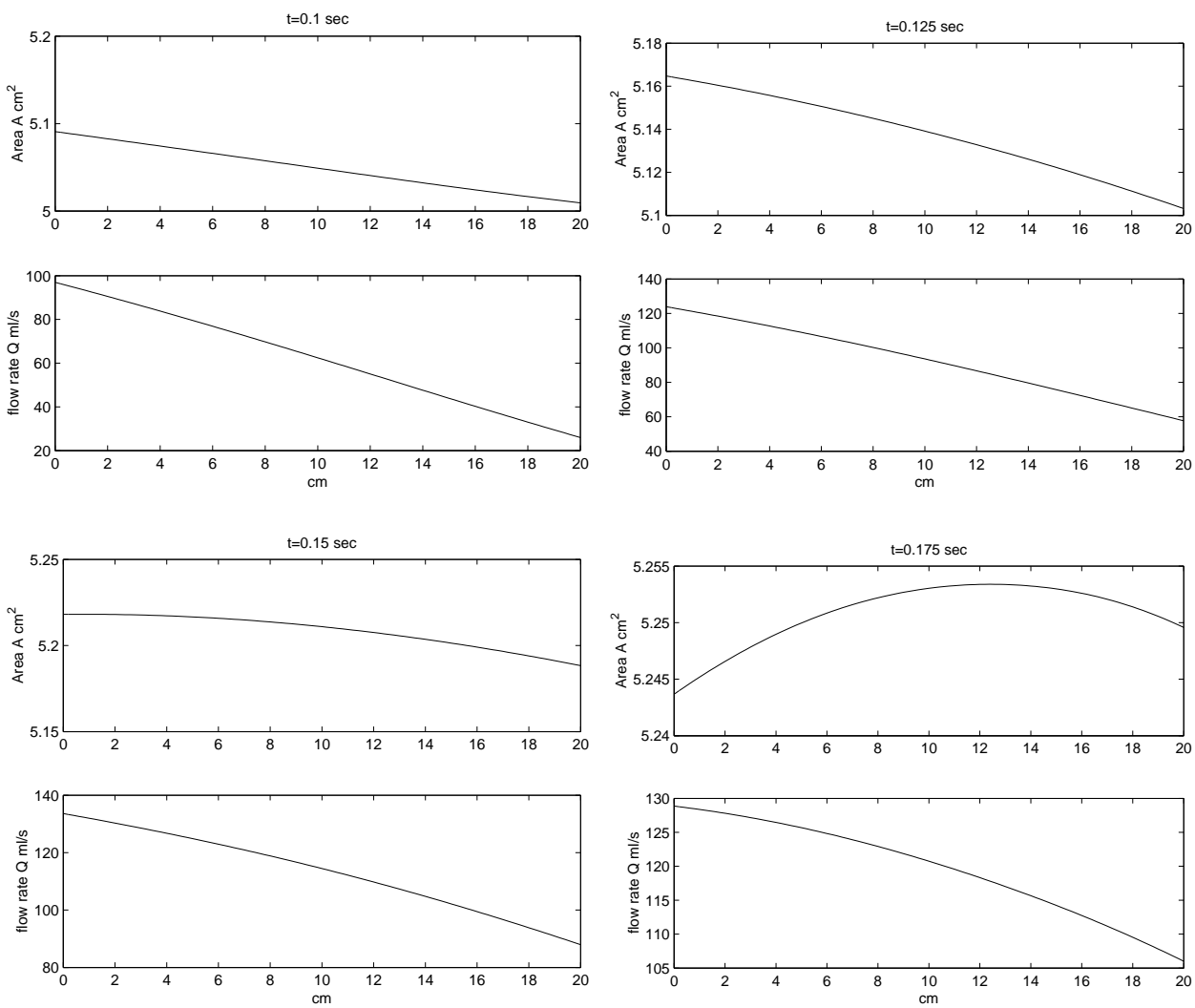

Figure 6: Snapshots of the solution of the 1D model at four time levels

the dotted-line represents the pure 0D model with the equivalent $R_{\mathrm{s} 21} L_{\mathrm{s} 21} C_{\mathrm{s} 21}$ (Resistance Inductance Compliance) network replacing the 1D part.

\section{Conclusion}

In this work, we have proved the existence and uniqueness of solutions of the coupled system (16). This is a general result whose interest goes beyond the specific case here described in which the coupled system represents a hybrid model of blood flow in a tract of the arterial tree. Indeed, it can be applied to every coupled problem involving a hyperbolic $2 \times 2$ system that can be written in diagonal form like (6) and a system of ODE's with locally Lipschitz right hand side. We point out that the hypotheses insuring the existence and uniqueness of our coupled problem are not restrictive for realistic applications to blood flow analysis. 

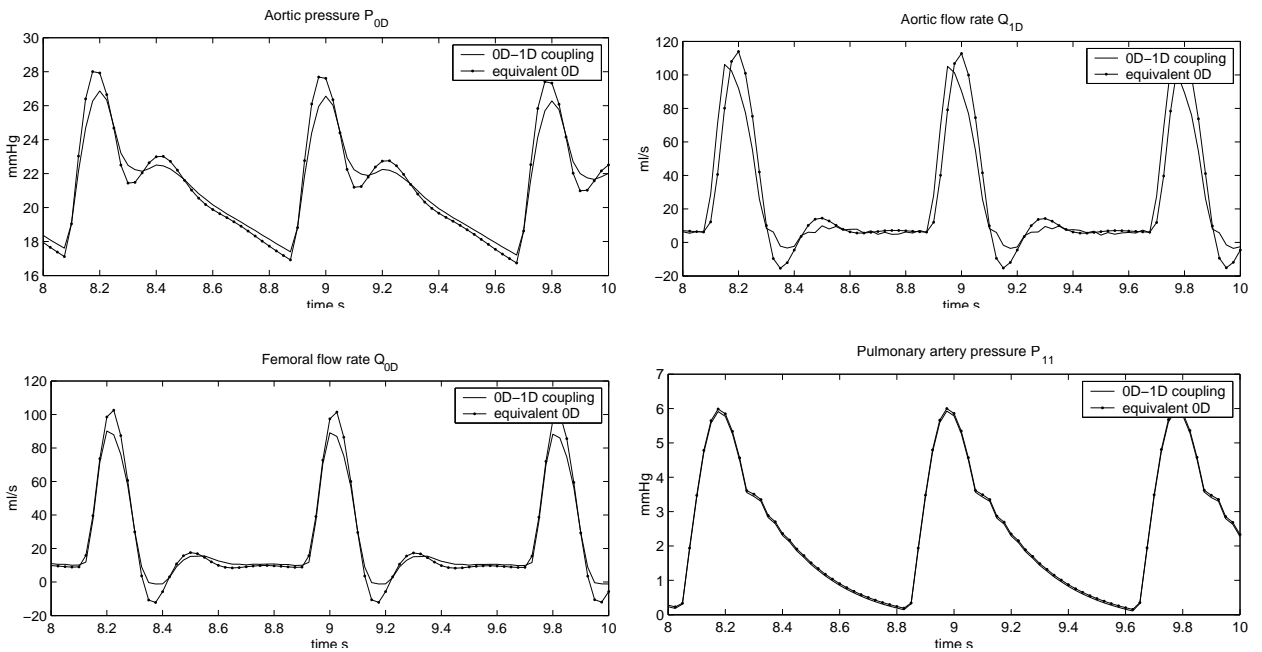

Figure 7: Pressure $P_{0 \mathrm{D}}$, flow rate $Q_{0 \mathrm{D}}$, pulmonary pressure $P_{11}$, and pulmonary flow rate $Q_{12}$

\begin{tabular}{||c|c|c|c|c||}
\hline $\begin{array}{c}\text { part } \\
\text { of the body }\end{array}$ & $\begin{array}{c}\text { variables } \\
\text { subindex }\end{array}$ & $\mathrm{R}$ & $\mathrm{L}$ & $\mathrm{C}$ \\
\hline arterial tree & $\mathrm{s} 1$ & $3.751 \cdot 10^{-3}$ & - & 0.6 \\
& $\mathrm{~s} 21$ & $5.47 \cdot 10^{-4}$ & $3.057 \cdot 10^{-3}$ & 0.2454 \\
& $\mathrm{~s} 22$ & 0.059453 & $1.943-3$ & 0.3546 \\
& $\mathrm{~s} 3$ & 0.05 & 0.6 & $0.1 \cdot 10^{-2}$ \\
\hline capillaries & $\mathrm{cap}$ & 0.83 & - & - \\
\hline veins & $\mathrm{vs}$ & 0.11 & - & 82.5 \\
\hline & 3 & $3.751 \cdot 10^{-3}$ & - & 20 \\
\hline lungs & 5 & $3.751 \cdot 10^{-3}$ & - & $9 \cdot 10^{-2}$ \\
& 6 & $3.376 \cdot 10^{-2}$ & $7.5 \cdot 10^{-4}$ & 2.67 \\
& 7 & 0.1013 & $3.08 \cdot 10^{-3}$ & 2.67 \\
& 8 & $3.751 \cdot 10^{-3}$ & - & - \\
\hline
\end{tabular}

Table 1: Table of coefficients used for the electric (0D) network, resistances are given in $m m H g \cdot s \cdot m l^{-1}$, inductances in $m m H g \cdot s^{2} \cdot m l^{-1}$, capacitances in $m l \cdot m m H^{-1}$

Further investigations related to our results will concern: stability and convergence analysis of the discrete coupled problem, the extension of the present results to the case of weak solutions (mandatory when introducing vascular prosthesis like a stent in a cardiovascular tree) $[18,12]$. 


\section{Acknowledgments}

The first author was supported by the Swiss National Science Foundation (contract number 20-65110.01). The second and third authors were supported by the European Union through the RTN project HaeMOdel (contract number HPRN-CT-2002-00270). The third author also acknowledges the contribution of the research project MLUR-Cofin 2003 "Advanced Numerical Methods for Applications of Partial Differential Equations" and INDAM. The authors thank Fabio Nobile for its contribution to numerical tests.

\section{References}

[1] G. Birkhoff and G.C. Rota. Ordinary differential equations. John Wiley \& Sons Inc., New York, fourth edition, 1989.

[2] S. Čanić and E.H. Kim. Mathematical analysis of quasilinear effects in a hyperbolic model of blood flow through compliant axi-symmetric vessels. Math. Meth. Appl. Sci., 26:1161-186, 2003.

[3] L. Formaggia, J. F. Gerbeau, F. Nobile, and A. Quarteroni. On the coupling of 3D and 1D Navier-Stokes equations for flow problems in compliant vessels. Comput. Methods Appl. Mech. Engrg., 191(6-7):561-582, 2001.

[4] L. Formaggia, D. Lamponi, M. Tuveri, and A. Veneziani. Numerical modelling of 1D arterial networks coupled with a lumped parameter description of the heart. Submitted.

[5] L. Formaggia, F. Nobile, and A. Quarteroni. A one dimensional model for blood flow: application to vascular prosthesis. In Mathematical modeling and numerical simulation in continuum mechanics (Yamaguchi, 2000), volume 19 of Lect. Notes Comput. Sci. Eng., pages 137-153. Springer, Berlin, 2002.

[6] L. Formaggia, F. Nobile, A. Quarteroni, and A. Veneziani. Mulstiscale modelling of the circulatory system: a preliminary analysis. Comput. Visual Sci., 2:75-83, 1999.

[7] L. Formaggia, F. Nobile, A. Veneziani, and A. Quarteroni. Multiscale modelling of the circulatory system: a preliminary analysis. Comput. Visual. Sci, 2:75-83, 1999.

[8] L. Formaggia and A. Veneziani. Reduced and multiscale models for the human cardiovascular system. 7th VKI Lecture Series on "Biological fluid dynamics", Von Karman Institute, Belgium, 2003.

[9] E. Godlewski and P.-A. Raviart. Hyperbolic systems of conservation laws, volume $3 / 4$ of Mathématiques $\&$ Applications (Paris) [Mathematics and Applications]. Ellipses, Paris, 1991.

[10] F.C. Hoppensteadt and C.S. Peskin. Mathematics in medicine and the life sciences, volume 10 of Texts in Applied Mathematics. Springer-Verlag, New York, 1992.

$\mathrm{RR} \mathrm{n}^{\circ} 5127$ 
[11] K. Laganà, G. Dubini, F. Migliavacca, R. Pietrabissa, G. Pennati, A. Veneziani, and A. Quarteroni. Multiscale modelling as a tool to prescribe realistic boundary conditions for the study of surgical procedures. Biorheology, 39:359-364, 2002.

[12] D. Lamponi, V. Milišić, and A. Quarteroni. Coupling 0D and 1D models for blood flow. In preparation.

[13] T.-T. Li. Global classical solutions for quasilinear hyperbolic systems. Masson, Paris, 1994.

[14] T.-T. Li and W.C. Yu. Boundary value problems for quasilinear hyperbolic systems. Duke University Mathematics Series, V. Duke University Mathematics Department, Durham, NC, 1985.

[15] S. Mantero, R. Pietrabissa, and R. Fumero. The coronary bed and its role in the cardiovascular system: a review and an introductory single-branch model. J. Biomed. Eng., 11:109-115, 1992.

[16] B.C. McInnis, Z.W. Guo, P.C. Lu, and J.C. Wang. Adaptive control of left ventricular bypass assist devices. IEEE Trans Autom. Control, 30:322-329, 1985.

[17] F. Migliavacca, G. Pennati, G. Dubini, R. Fumero, R. Pietrabissa, G. Urcelay, E.L. Bove, T.Y. Hsia, and M. De Leval. Modeling of the norwood circulation: effects of shunt size, vascular resistances, and heart rate. Am. J. Physiol. Heart Circ. Physiol., 280:2076-2086, 2001.

[18] V. Milišić and A. Quarteroni. New consistency and convergence analysis of lumped parameter models for blood flow simulations. In preparation.

[19] M.S. Olufsen, C.S. Peskin, Kim. W.Y., E.M. Pedersen, A. Nadim, and J. Larsen. Numerical simulation and experimental validation of blood flow in arteries with structuredtree outflow conditions. Annals of Biomedical Engineering, 28:1281-1299, 2000.

[20] K. Perktold and G. Rappitsch. Mathematical modeling of local arterial flow and vessel mechanics. In Computational methods for fluid-structure interaction (Les Moussières/Métabief, 1992), volume 306 of Pitman Res. Notes Math. Ser., pages 230-245. Longman Sci. Tech., Harlow, 1994.

[21] A. Quarteroni, S. Ragni, and A. Veneziani. Coupling between lumped and distributed models for blood flow problems. Comput. Visual Sci., 4:111-124, 2001.

[22] A. Quarteroni, M. Tuveri, and A. Veneziani. Computational vascular fluid dynamics: problems, models and methods. Comput. Visual Sci., 2:163-197, 2000.

[23] A. Quarteroni and A. Valli. Numerical approximation of partial differential equations, volume 23 of Springer Series in Computational Mathematics. Springer-Verlag, Berlin, 1994. 
[24] A. Quarteroni and A. Veneziani. Analysis of a geometrical multiscale model based on the coupling of pde's and ode's for blood flow simulations. SIAM J. on Multiscale Model. Simul., 1(2):173-195, 2003.

[25] K. Sagawa, H. Suga, and Nakayama K. Instantaneous pressure-volume ratio of the ventricle versus instantaneous force-length relation of papillary muscle, pages 99-105. Cardiovascular System Dynamics. MIT Press, Cambridge, Massachusetts, 1978.

[26] D. Serre. Systèmes de lois de conservation. II. Diderot Editeur, Paris, 1996.

[27] S. Sherwin, L. Formaggia, J. Peiró, and V. Franke. Computational modelling of 1d blood flow with variable mechanical properties and its application to the simulation of wave propagation in the human arterial system. To appear in Int. J. Numer. Meth. Fluids (2003).

[28] S. Sherwin, V. Franke, J. Peiró, and K. Parker. One-dimensional modelling of a vascular network in space-time variables. Submited to Journal of Engineering Mathematics.

[29] N.P. Smith, A.J. Pullan, and P.J. Hunter. An anatomically based model of transient coronary blood flow in the heart. SIAM J. Appl. Math., 62(3):990-1018, 2001/02.

[30] N. Stergiopulos, D.F. Young, and T.R. Rogge. Computer simulation of arterial flow with applications to arterial and aortic stenoses. J. Biomech., 25:1477-1488, 1992.

[31] N. Westerhof, F. Bosman, C.J. De Vries, and A. Noordergraaf. Analog studies of the human systemic arterial tree. J. Biomechanics, 2:121-143, 1969.

[32] E. Zeidler. Applied functional analysis, volume 109 of Applied Mathematical Sciences. Springer-Verlag, New York, 1995. 


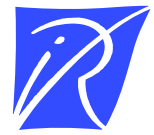

Unité de recherche INRIA Rocquencourt

Domaine de Voluceau - Rocquencourt - BP 105 - 78153 Le Chesnay Cedex (France)

Unité de recherche INRIA Futurs : Parc Club Orsay Université - ZAC des Vignes

4, rue Jacques Monod - 91893 ORSAY Cedex (France)

Unité de recherche INRIA Lorraine : LORIA, Technopôle de Nancy-Brabois - Campus scientifique

615, rue du Jardin Botanique - BP 101 - 54602 Villers-lès-Nancy Cedex (France)

Unité de recherche INRIA Rennes : IRISA, Campus universitaire de Beaulieu - 35042 Rennes Cedex (France)

Unité de recherche INRIA Rhône-Alpes : 655, avenue de l'Europe - 38334 Montbonnot Saint-Ismier (France)

Unité de recherche INRIA Sophia Antipolis : 2004, route des Lucioles - BP 93 - 06902 Sophia Antipolis Cedex (France) 